\title{
Malaria preventive therapy in pregnancy and its potential impact on immunity to malaria in an area of declining transmission
}

\author{
Andrew Teo ${ }^{1 *}$, Wina Hasang ${ }^{1,2}$, Louise M. Randall ${ }^{1,2}$, Holger W. Unger ${ }^{1}$, Peter M. Siba ${ }^{3}$, Ivo Mueller ${ }^{4,5}$,
} Graham V. Brown ${ }^{1,2,6}$ and Stephen J. Rogerson ${ }^{1,2}$

\begin{abstract}
Background: Regular anti-malarial therapy in pregnancy, a pillar of malaria control, may affect malaria immunity, with therapeutic implications in regions of reducing transmission.

Methods: Plasma antibodies to leading vaccine candidate merozoite antigens and opsonizing antibodies to endothelial-binding and placental-binding infected erythrocytes were quantified in pregnant Melanesian women receiving sulfadoxine-pyrimethamine (SP) with chloroquine taken once, or three courses of SP with azithromycin.

Results: Malaria prevalence was low. Between enrolment and delivery, antibodies to recombinant antigens declined in both groups $(p<0.0001)$. In contrast, median levels of opsonizing antibodies did not change, although levels for some individuals changed significantly. In multivariate analysis, the malaria prevention regimen did not influence antibody levels.
\end{abstract}

Conclusion: Different preventive anti-malarial chemotherapy regimens used during pregnancy had limited impact on malarial-immunity in a low-transmission region of Papua New Guinea.

Trial registrations: NCT01136850

Keywords: Malaria, Antibody, Papua New Guinea, Pregnancy, Immunity, Azithromycin, Sulfadoxine-pyrimethamine, Chloroquine, IPTp, ITN

\section{Background}

Pregnant women are susceptible to Plasmodium falciparum infection, and malaria in pregnancy (MiP) increases the risks for mother and baby [1]. Antibody to placentalbinding infected erythrocytes (IEs) can protect against $\mathrm{MiP}$ and its consequences [2, 3]. Intermittent preventive therapy during pregnancy (IPTp) and insecticide-treated bed nets (ITN) can reduce the impact of MiP [4], but could impair development of pregnancy-associated immunity [5]. Current data on the impact of malaria prevention on the acquisition of pregnancy-associated malarial antibodies is largely restricted to African settings. It was hypothesized that multiple courses of IPTp during pregnancy may be associated with impaired development of

\footnotetext{
* Correspondence: andrew.teo@uqconnect.edu.au

'University of Melbourne, Department of Medicine (Royal Melbourne Hospital), Melbourne, VIC, Australia

Full list of author information is available at the end of the article
}

pregnancy-associated malaria immunity, and this was tested in a cohort of pregnant women from Madang, Papua New Guinea (PNG).

\section{Methods}

Ethics approval

Ethics approval was obtained from the PNG Institute of Medical Research's Institutional Review Board (08.15), the PNG Medical Research Advisory Council (05.03, 10.50) and the Human Research Ethics Committee of Melbourne Health (2001.016, 2008.162). Women provided written informed consent.

\section{Study participants}

Pregnant women were recruited for a malaria prevention trial in Madang, PNG, and plasma samples from a subset of these women were used in this study [6]. Participants recruited at first antenatal visit (ANC) were 
randomly assigned to receive one course of sulfadoxinepyrimethamine (SP) with chloroquine (CQ) $(N=304)$, control arm, or up to three courses of SP and azithromycin (AZ) $(N=277)$, intervention arm, and followed to delivery. Women were given an ITN, if available, and usage was recorded. Paired plasma samples collected at enrolment and delivery were assayed for $P$. falciparum antibodies.

\section{Malariometric indices}

Presence of P. falciparum infection (enrolment, delivery) was determined by light microscopy (LMS) and quantitative polymerase chain reaction (qPCR) of peripheral blood films, from placental impression (LMS, qPCR), and by examination of placental histology. Placental malaria was classified as acute, chronic or past infection [7]

\section{Parasite and cell cultures}

The laboratory-adapted $P$. falciparum lines CS2 (placentalbinding) and E8B-ICAM (endothelial-binding), and THP-1 monocyte-like cells, were cultured as described [8].

\section{Assays of IgG to schizont extract, merozoite antigens and measles haemagglutinin}

Samples were assayed for immunoglobulin G (IgG) antibodies to recombinant $P$. falciparum antigens and measles haemagglutinin protein by enzyme-linked immunosorbent assay (ELISA) as described [8]. In brief, microtitre plates were coated with schizont extract from CS2 (1/2000), MSP2 from FC27 $(0.5 \mu \mathrm{g} / \mathrm{ml})$, MSP3 from 3D7 full ectodomain $(2 \mu \mathrm{g} / \mathrm{ml})$, PfRH2 from 3D7 $(0.5 \mu \mathrm{g} / \mathrm{ml})$ and measles haemagglutinin $(1 \mu \mathrm{g} / \mathrm{ml}$; Abcam, Melbourne, VIC, Australia). Test plasma (1/1000, in duplicate) was added, followed by incubation with peroxidase-conjugated goat anti-human IgG (1/2500; Merck Millipore, Kilsyth, VIC, Australia). The reaction was developed and optical density was determined at $405 \mathrm{~nm}$.

\section{Phagocytosis of infected erythrocytes}

The level of opsonizing IgG antibody was determined as before [8]. In brief, $30 \mu \mathrm{L}$ of purified trophozoite-stage IEs were stained with ethidium bromide, and opsonized with $3.3 \mu \mathrm{l}$ of plasma for $1 \mathrm{~h}$, followed by incubation with THP-1 cells for 40 min. Phagocytosis was stopped and unphagocytosed IEs were lysed, followed by fixing the THP-1 cells in $2 \%(w / v)$ paraformaldehyde. The cells were acquired using a HyperCyt ${ }^{\circ}$ CyAn flow cytometer (Beckman Coulter). Data are represented as percentage of THP-1 cells containing ingested IEs. For all assays, plasma from six adults with no history of malaria exposure was included. Discordant samples were re-run, using published rules [9].

\section{Antibody responses during pregnancy}

To assess changes during pregnancy, the mean antibody levels from paired samples collected at enrolment and delivery were compared, and the same rules were applied as described [8]. In brief, samples were categorized based on their adjusted mean variance: 1) $<20 \%$ with mean difference of $<10 \%$, no change; 2) $>20 \%$ with mean difference of $>10 \%$, either decrease or increase in antibody responses.

\section{Statistical analysis}

The main interest of analysis is whether preventive therapies during pregnancy had an impact on development of pregnancy related anti-malarial antibodies. Data were analysed with Stata version 13.0 (StataCorp) and GraphPad Prism v5 (GraphPad Software, Inc). Differences in population mean ranks of paired continuous non-parametric variables, antibody responses, were evaluated using the Wilcoxon signed-rank test. Categorical variables were compared using the $\chi^{2}$ tests. Multiple linear regression analysis was performed to determine associations between continuous and categorical variables on antibody, and adjusted analyses included treatment arms, maternal characteristics, and interaction variables.

\section{Results}

\section{Patient characteristics}

Participant characteristics (SP-CQ $n=304$, SP-AZ $n=277$ ) were similar between groups. Most women receiving SP-AZ (82 \%) had three courses. The prevalence of malaria infection was low at enrolment and delivery (Table 1).

\section{Antibody to recombinant antigens}

Median antibody levels to schizont extract and merozoite antigens did not differ by treatment arm at delivery (schizont extract $\mathrm{z}=-0.4, \operatorname{PfRh} 2 \mathrm{z}=-0.8, \operatorname{MSP} 2 \mathrm{z}=0.2$, MSP3 $\mathrm{z}=-0.3$, all $p>0.05$ ). There was a significant decline in median level of antibodies against schizont extract, MSP2, MSP3 and PfRh2 between enrolment and delivery (all $p<0.0001$ ) in both treatment arms. Antibody to measles haemagglutinin (control) showed a similar significant decline in both treatment arms (Fig. 1a).

\section{Opsonizing antibody to infected erythrocytes}

Opsonizing antibodies to placental-binding and-endothelialbinding IEs did not vary by treatment arm at delivery $($ CS2 $\mathrm{z}=-0.8$, E8B-ICAM $\mathrm{z}=0.6$, both $p>0.05)$. In contrast to decline in antibodies to recombinant antigens (above), median levels of opsonizing antibodies to both placental-binding and-endothelial-binding IEs did not change significantly between enrolment and delivery in either treatment arm (Fig. 1b). 
Table 1 Study population characteristics of Papua New Guinean women

\begin{tabular}{|c|c|c|c|}
\hline Characteristic & $\begin{array}{l}\text { Single course } \\
\text { of CQ and SP } \\
\text { treatment } \\
(n=304)\end{array}$ & $\begin{array}{l}\text { Three courses } \\
\text { of SP and AZ } \\
\text { treatment } \\
(n=277)\end{array}$ & $P$-value \\
\hline Age, years & $24.0(21.0-28.0)$ & $24.0(21.0-28.0)$ & 0.2 \\
\hline Weight at enrolment, kg & $55.0(50.0-59.0)$ & $53.0(49.0-59.0)$ & 0.6 \\
\hline MUAC at enrolment, $\mathrm{cm}$ & $23.0(22.0-25.0)$ & $23.0(22.0-25.0)$ & 0.5 \\
\hline Gravidity & & & 0.4 \\
\hline Gravida 1, n (\%) & $108(35.5)$ & $92(33.2)$ & \\
\hline Gravida 2, n (\%) & $91(29.9)$ & $88(31.8)$ & \\
\hline Gravida 3, n (\%) & $105(34.5)$ & $97(35.0)$ & \\
\hline Overall Bed net use & & & 0.4 \\
\hline No, $n(\%)$ & $2(0.7)$ & $0(0.0)$ & \\
\hline Intermittent, $n(\%)$ & $89(29.3)$ & $82(29.6)$ & \\
\hline Regular, $n(\%)$ & $213(70.1)$ & $195(70.4)$ & \\
\hline Ethnicity & & & 0.5 \\
\hline Madang/Morobe & $194(63.8)$ & $193(69.7)$ & \\
\hline Sepik & $57(18.8)$ & $41(14.8)$ & \\
\hline Highland & $26(8.6)$ & $19(6.9)$ & \\
\hline Others & $27(8.9)$ & $24(8.7)$ & \\
\hline Residence & & & 0.9 \\
\hline Urban & $51(16.8)$ & $44(16.0)$ & \\
\hline Peri-urban & $59(19.5)$ & $50(18.1)$ & \\
\hline Rural & $181(59.7)$ & $170(61.8)$ & \\
\hline Migrant & $12(4.0)$ & $11(4.0)$ & \\
\hline \multicolumn{4}{|l|}{$\begin{array}{l}\text { Light microscopy }(P . \\
\text { falciparum) }\end{array}$} \\
\hline Enrolment (peripheral blood) & $20(6.6)$ & $15(5.4)$ & 0.5 \\
\hline Delivery (peripheral blood) & $7(2.3)$ & $5(1.8)$ & 0.7 \\
\hline Delivery (placental blood) & $7(2.3)$ & $4(1.4)$ & 0.2 \\
\hline \multicolumn{4}{|l|}{ qPCR (P. falciparum) } \\
\hline Enrolment (peripheral blood) & $31(10.2)$ & $24(8.7)$ & 0.8 \\
\hline Delivery (peripheral blood) & $14(4.6)$ & $8(2.9)$ & 0.1 \\
\hline Delivery (placental blood) & $7(2.3)$ & $1(0.3)$ & 0.1 \\
\hline Placental histology & & & 0.1 \\
\hline Uninfected & $171(81.8)$ & $170(81.0)$ & \\
\hline Infected ${ }^{\mathrm{a}}$ & $38(18.2)$ & $40(19.1)$ & \\
\hline
\end{tabular}

Data represented as median and interquartile range, unless otherwise indicated $A Z$ azithromycin, $C Q$ chloroquine, $S P$ sulfadoxine-pyrimethamine, MUAC mid-upper arm circumference

${ }^{\text {a }}$ Placental malaria was defined as histological evidence of acute, chronic, or past infection

\section{Changes in individual antibody responses by treatment arm between enrolment and delivery}

When differences in individual responses were compared between enrolment and delivery, for each treatment arm, similar numbers of women experienced changes in antibodies to schizont extract, MSP2 and MSP3. Significantly more women on SP-CQ than SP-AZ experienced an increase in antibody response to PfRh2 ( 8.3 vs $3.6 \% ; p=0.05$ ), and in opsonizing antibody to endothelial-binding IEs (29.6 vs $19.1 \%$; $p=0.004)$. Interestingly, significantly more women on SP-AZ than SP-CQ experienced an increase in opsonizing antibody to placental-binding IEs (17.8 vs $12.0 \%$; $p=0.03$; Table 2 ).

\section{Effect of treatment arm on antibodies at delivery: adjusted analysis}

After adjusting for confounding and interaction variables, there were no significant differences in antibody levels at delivery between treatment arms (Table 3). Antibody levels at delivery were associated with rural residence (for schizont extract, MSP2 and MSP3), and with highlander heritage (schizont extract and MSP2), all $p<0.05$. There was also a gravidity-dependent increase in opsonizing antibody to both endothelial-binding and-placental-binding IEs, both $p<0.01$. Importantly, there was no evidence of the intervention arm modifying levels of malarial antibodies in higher gravidity women and women from different areas and ethnicity.

\section{Discussion}

Malaria prevention during pregnancy reduces exposure and may affect the acquisition of malaria immunity $[4,5]$. In these PNG women, levels of antibody to merozoite and schizont antigens declined during pregnancy whether women received single dose of SP-CQ or multiple doses of SP-AZ.

The reduction in median levels of antibody to schizont extract and several recombinant merozoite antigens between enrolment and delivery may be due to reduced exposure and/or effective clearance and protection by SP-AZ or SP-CQ and ITN. Other possible contributions include the low prevalence of infection and maternal haemodilution, as indicated by a concomitant decline in antibody to measles haemagglutinin. In contrast, there were no significant differences in median levels of opsonizing antibodies to placental-binding IEs or to endothelialbinding IEs. These observations are consistent with a previous study from PNG demonstrating maintenance of opsonizing antibodies during lower force of infection [8]. Antibodies to merozoite antigens may have a shorter half-life than antibodies to IEs [10]. This could explain differences observed, if repeated exposure is required to maintain anti-merozoite antibodies.

There are many different assays for measuring potentially protective anti-malarial antibody. In Ghana, antibodies to recombinant placental-binding P. falciparum erythrocyte membrane protein 1 (PfEMP1) proteins increased during pregnancy and declined after delivery, suggesting that these antibody responses are transient in 

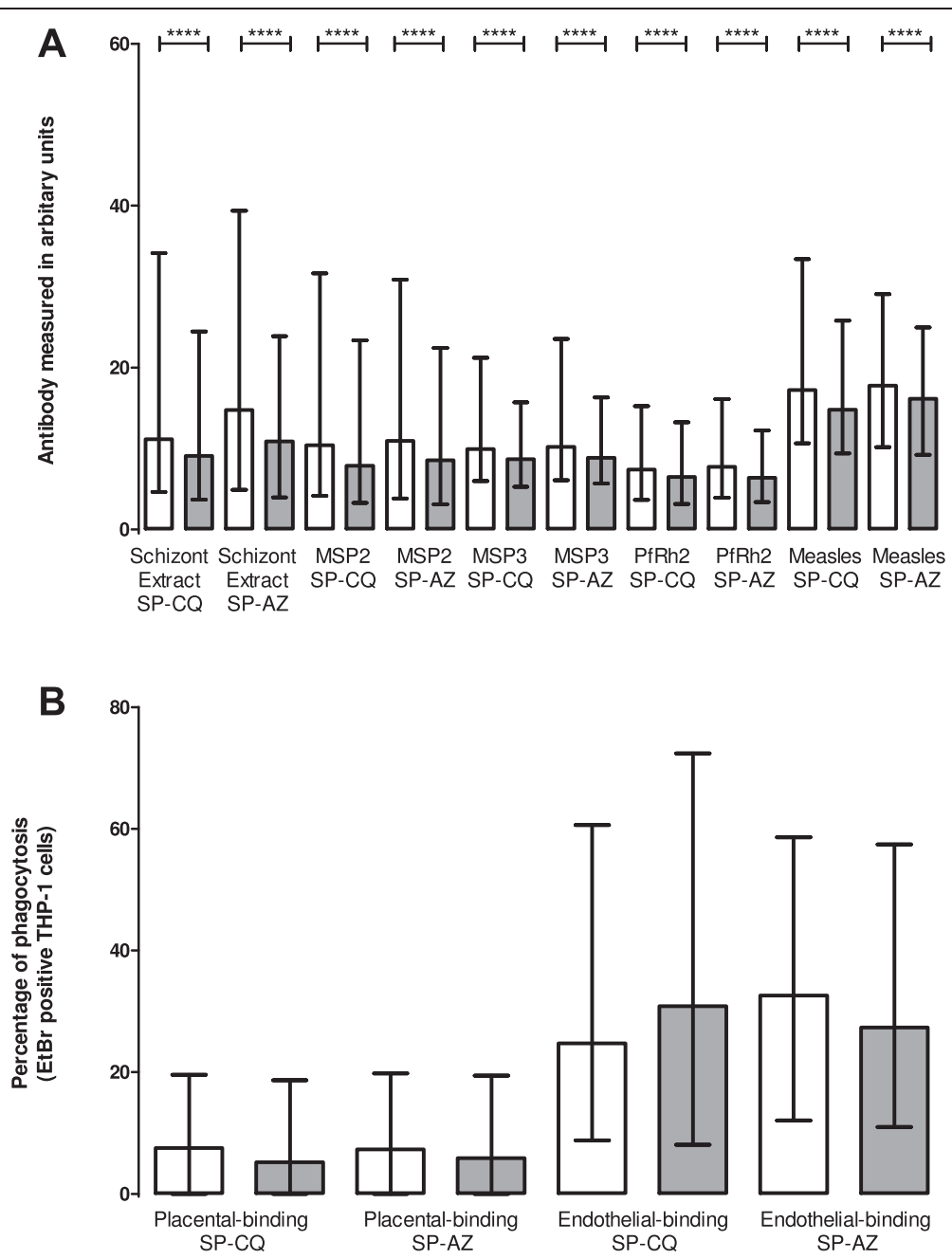

Fig. 1 Levels of immunoglobulin G (IgG) antibody in PNG pregnant women against Plasmodium falciparum antigens over the course of one pregnancy. White bars- pregnant women recruited at first antenatal visit, grey bars pregnant women at delivery. Pregnant women on sulfadoxine-pyrimethamine (SP) and chloroquine (CQ) [N=304], and on SP and azithromycin (AZ) [N=277]. a Levels of IgG antibodies to schizont extract, PfRh2, MSP2, MSP3 and measles antigen presented as arbitrary units. $\mathbf{b}$ Levels of opsonising $\operatorname{lgG}$ antibodies to variant surface antigens of placental-binding and endothelialbinding IEs, presented as percentage of THP-1 cells that have ingested IESs (percentage phagocytosis). Wilcoxon signed-rank test, ${ }^{* * * *} p<0.0001$. Columns represents IQR and error bars shows $95 \% \mathrm{Cl}$

the absence of infection [11]. The lack of rise in opsonizing antibodies to placental-binding IEs may reflect the lower force of infection in this study, but lack of decline in these circumstances suggests that opsonizing antibodies might be more stable than IgG to recombinant antigens [8]. In Ghana, the levels of antibodies to recombinant endothelial-binding PfEMP1 proteins did not vary during pregnancy [11], similar to the present findings for opsonizing antibodies to endothelial-binding IEs.

The relative stability of functional opsonizing antibodies even with low force of infection, compared with IgG directed to recombinant antigens, makes their levels a potential measure of longer lasting immunity to MiP. Whether functional properties of antibodies to IEs, including opsonizing activity and inhibition of placental binding [2] are the best correlates of protective immunity requires further exploration. Longitudinal studies of functional antibodies to merozoites [12] during pregnancy would also be of great interest.

Anti-malarial prevention strategies may affect the development of pregnancy-associated malaria immunity [5, 13], but in the present study the change in opsonizing antibody to placental-binding IEs did not differ between treatment arms, after controlling for confounders and explanatory variables. The disparity between the current study and others may be explained by the differences in endemicity, high ITN coverage in this cohort, and differences in assays used. Malaria antibodies vary during pregnancy $[9,11]$, and it was observed that analysis at group level concealed heterogeneity in responses among 
Table 2 Changes in antibody responses against $P$. falciparum antigens in the Papua New Guinean cohort during the course of pregnancy

\begin{tabular}{|c|c|c|c|}
\hline Variable $(n)$ & $\begin{array}{l}\text { SP + CQ control } \\
\text { arm }(n=304)\end{array}$ & $\begin{array}{l}\mathrm{SP}+\mathrm{AZ} \text { intervention } \\
\mathrm{arm}(n=277)\end{array}$ & $P$ value \\
\hline IgG to schizont extract & & & 0.7 \\
\hline Decrease, $n(\%)$ & $70(23.2)$ & $67(24.3)$ & \\
\hline No change, $n(\%)$ & $198(65.6)$ & $184(66.8)$ & \\
\hline Increase, $n(\%)$ & $34(11.3)$ & $25(9.1)$ & \\
\hline $\lg G$ to MSP2 & & & 0.6 \\
\hline Decrease, $n(\%)$ & $63(20.9)$ & $49(17.8)$ & \\
\hline No change, $n(\%)$ & $217(71.9)$ & $203(73.6)$ & \\
\hline Increase, $n(\%)$ & $22(7.3)$ & $24(8.7)$ & \\
\hline $\operatorname{lgG}$ to MSP3 & & & 0.8 \\
\hline Decrease, $n(\%)$ & $55(18.2)$ & $53(19.1)$ & \\
\hline No change, $n(\%)$ & $220(72.8)$ & $203(73.3)$ & \\
\hline Increase, $n(\%)$ & $27(8.9)$ & $21(7.6)$ & \\
\hline $\operatorname{lgG}$ to PfRh2 & & & 0.05 \\
\hline Decrease, $n(\%)$ & $34(11.2)$ & $39(14.1)$ & \\
\hline No change, $n(\%)$ & $244(80.5)$ & $228(82.3)$ & \\
\hline Increase, $n(\%)$ & $25(8.3)$ & $10(3.6)$ & \\
\hline $\begin{array}{l}\text { Opsonizing lgG to } \\
\text { E8B-ICAM }\end{array}$ & & & 0.004 \\
\hline Decrease, $n(\%)$ & $72(23.9)$ & $60(21.7)$ & \\
\hline No change, $n(\%)$ & $140(46.5)$ & $164(59.2)$ & \\
\hline Increase, $n(\%)$ & $89(29.6)$ & $53(19.1)$ & \\
\hline Opsonizing lgG to CS2 & & & 0.03 \\
\hline Decrease, $n(\%)$ & $39(13.0)$ & $47(17.0)$ & \\
\hline No change, $n(\%)$ & $226(75.1)$ & $180(65.2)$ & \\
\hline Increase, n (\%) & $36(12.0)$ & $49(17.8)$ & \\
\hline
\end{tabular}

Data represented as numbers and percentage, $P$-values are also shown $A Z$ azithromycin, $C Q$ chloroquine, $S P$ sulfadoxine-pyrimethamine Significant associations $(p<0.05)$ highlighted in bold

individuals. For some antigens, the variations in individual women's antibody responses between enrolment and delivery differed by treatment arm. A greater proportion of women on SP-CQ experienced increases in antibody to PfRh2 and in opsonizing antibody against endothelial-binding IEs than women on SP-AZ, consistent with increased exposure associated with the less effective intervention. Supporting this, in the parent study SP-AZ decreased parasite prevalence at delivery by microscopy and placental histology [14]. Although multiple courses of anti-malarials during pregnancy may decrease malaria exposure, the impact on immunity in the context of low prevalence of infection and high ITN use appears modest.

In contrast to antibody to endothelial-binding IEs, levels of opsonizing antibodies to placental-binding IEs were more likely to increase or decrease (rather than remaining constant) in the SP-AZ arm compared with the SP-CQ arm, consistent with previous observations [8]. This reflects the complex dynamics of pregnancy-specific malarial immunity during a single pregnancy [9-11]. Assaying at multiple time points during pregnancy may assist better evaluation of pregnancy-associated immune status.

In the adjusted analyses, factors influencing malarial antibody levels at delivery did not vary between the treatment arms. Increasing gravidity is associated with higher opsonizing antibodies to IEs consistent with graviditydependent acquisition of pregnancy-specific immunity [2]. Rural women had higher antibodies to schizont extract, MSP2 and MSP3 than women from urban areas, whereas highlands-born women tended to have lower antibody levels to schizont extract and MSP2. These differences, which are indicative of more malaria exposure with increasing gravidity and in women residing in the rural areas, and lower exposure in the highlands-born women, were independent of treatment arm. Anti-malarials could have a greater impact on malaria immunity in settings of lower ITN usage and higher prevalence of infection.

The current study may have underestimated potential benefits of prevention. First, the low malaria prevalence may have reduced the power to observe any potential impact of different treatment arms on the acquisition of pregnancy-associated malaria immunity. Second, although high ITN coverage may reduce the need for intensive IPTp and, consequently, reduce selection pressure on the parasites $[15,16]$, high ITN coverage may have further reduced malaria exposure in this study, obscuring a possible effect of drug regimen on immune responses. Finally, the time for which women participated varied between three and six months; earlier implementation of malaria prevention might have had a greater impact on immunity development. Studies from higher-transmission areas, spanning most or all of gestation, are more likely to demonstrate the impact of malaria prevention on the acquisition of pregnancy-associated malaria immunity.

\section{Conclusions}

In conclusion, IPTp with multi-doses of SP-AZ or a single-dose SP-CQ had similar impacts on pregnancyassociated malaria immunity, possibly due to the low prevalence of infection and extensive ITN coverage observed. The decline in merozoite and schizont antibodies observed between enrolment and delivery supports this. This study also demonstrated an apparent maintenance of functional opsonizing antibodies, thought to be important for prevention of morbidity, which deserves further evaluation in other regions and in the context of other preventive measures to elucidate the optimum policy in preventing $\mathrm{MiP}$ in such regions. 
Table 3 Relative antibody responses against $P$. falciparum antigens at delivery by treatment arm in Madang, PNG, adjusted for confounding and interaction variables

\begin{tabular}{|c|c|c|c|c|c|c|c|c|c|c|c|c|}
\hline \multirow[t]{2}{*}{ Variables } & \multicolumn{2}{|l|}{ lgG schizont extract } & \multicolumn{2}{|l|}{$\operatorname{lgG}$ PfRh2 } & \multicolumn{2}{|l|}{$\operatorname{lgG}$ MSP2 } & \multicolumn{2}{|l|}{$\operatorname{lgG}$ MSP3 } & \multicolumn{2}{|c|}{ Opsonizing IgG E8B-ICAM } & \multicolumn{2}{|l|}{ Opsonizing lgG CS2 } \\
\hline & Coeff (95 \% Cl) & p & Coeff $(95 \%$ Cl) & p & Coeff $(95 \%$ Cl) & p & Coeff $(95 \%$ Cl) & $p$ & Coeff (95 \% Cl) & $p$ & Coeff $(95 \%$ Cl) & p \\
\hline Intervention arm $^{\mathrm{a}}$ & $-3.0(-13.4,7.4)$ & 0.6 & $-3.6(-10.9,3.7)$ & 0.3 & $-7.4(-17.9,3.1)$ & 0.2 & $2.6(-7.0,12.2)$ & 0.6 & $1.0(-14.2,16.2)$ & & $-7.4(-19.4,4.5)$ & 0.2 \\
\hline \multicolumn{13}{|l|}{ Gravidity } \\
\hline \multicolumn{13}{|l|}{1} \\
\hline 2 & $-2.0(-8.3,4.3)$ & 0.5 & $-4.6(-9.1,0.2)$ & 0.06 & $1.0(-5.44,7.42)$ & 0.8 & $-0.7(-6.6,5.1)$ & 0.8 & $50.3(42.8,57.9)$ & $<0.0001$ & $9.3(2.5,16.1)$ & 0.007 \\
\hline 3 & $1.6(-4.5,7.6)$ & 0.6 & $-0.5(-4.8,3.8)$ & 0.8 & $1.9(-4.3,8.1)$ & 0.6 & $5.72(-0.04,11.4)$ & 0.06 & $32.8(25.6,40.1)$ & $<0.0001$ & $24.3(17.9,30.8)$ & $<0.0001$ \\
\hline \multicolumn{13}{|c|}{ Overall bed net use } \\
\hline \multicolumn{13}{|l|}{ No } \\
\hline Intermittent & $-1.8(-6.0,2.4)$ & 0.4 & $-0.5(-3.4,2.5)$ & 0.8 & $-1.6(-5.9,2.7)$ & 0.5 & $0.3(-5.6,10.5)$ & 0.9 & $1.2(-3.8,6.2)$ & 0.6 & $-2.6(-7.2,1.9)$ & 0.3 \\
\hline Regular & $-13.0(-45.2,19.2)$ & 0.4 & $-5.3(-28.1,17.6)$ & 0.7 & $-12.0(-45.0,20.9)$ & 0.5 & $-2.5(-32.7,27.6)$ & 0.9 & $25.4(-13.1,63.9)$ & 0.2 & $-10.6(-45.0,23.8)$ & 0.6 \\
\hline \multicolumn{13}{|l|}{ Residence } \\
\hline \multicolumn{13}{|l|}{ Urban } \\
\hline Peri-urban & $0.3(-8.4,8.8)$ & 0.9 & $-1.3(-7.1,5.9)$ & 0.7 & $1.5(-7.2,10.2)$ & 0.7 & $2.0(-5.9,10.0)$ & 0.6 & $2.2(-10.4,14.9)$ & 0.4 & $-1.9(-11.8,7.9)$ & 0.7 \\
\hline Rural & $8.6(1.2,15.9)$ & 0.02 & $5.0(-0.2,10.0)$ & 0.06 & $8.5(1.2,16.0)$ & 0.02 & $8.5(1.7,15.2)$ & 0.01 & $-4.1(-14.8,6.6)$ & 0.7 & $-0.4(-8.8,8.0)$ & 0.9 \\
\hline Migrant & $13.0(-1.3,27.3)$ & 0.07 & $-0.1(-10.1,10.0)$ & 0.9 & $13.3(-1.1,27.7)$ & 0.07 & $-1.1(-14.3,12.1)$ & 0.9 & $6.4(-14.4,27.3)$ & 0.5 & $2.7(-13.7,19.1)$ & 0.8 \\
\hline \multicolumn{13}{|l|}{ Ethnicity } \\
\hline \multicolumn{13}{|l|}{ Madang/Morobe } \\
\hline Sepik & $-4.7(-11.0,11.0)$ & 0.2 & $0.3(-4.7,5.2)$ & 0.9 & $-5.4(-12.6,1.7)$ & 0.1 & $1.7(-4.8,8.2)$ & 0.6 & $-1.6(-11.9,8.7)$ & 0.8 & $4.7(-12.8,3.4)$ & 0.2 \\
\hline Highlands & $-12.3(-21.9,-2.6)$ & 0.01 & $-0.5(-7.2,6.2)$ & 0.9 & $-10.9(-20.6,-1.2)$ & 0.03 & $-1.0(-9.9,7.7)$ & 0.82 & $-3.7(-17.7,10.4)$ & 0.8 & $-6.4(-17.4,4.7)$ & 0.4 \\
\hline Others & $-4.4(-13.9,5.2)$ & 0.4 & $-2.5(-9.3,4.2)$ & 0.5 & $-7.5(-17.0,2.2)$ & 0.1 & $1.3(-7.5,10.1)$ & 0.8 & $-2.4(-16.5,11.7)$ & 0.9 & $-2.6(13.5,8.3)$ & 0.6 \\
\hline \multicolumn{13}{|l|}{ SP-AZ-gravidity ${ }^{a}$} \\
\hline \multicolumn{13}{|l|}{1} \\
\hline 2 & $-5.6(-14.77,3.56)$ & 0.2 & $7.1(0.57,-13.56)$ & 0.1 & $-6.5(-15.90,2.87)$ & 0.2 & $-3.5(-12.10,5.06)$ & 0.4 & $-6.5(-17.48,4.50)$ & 0.3 & $-0.3(-10.1,9.5)$ & 1.0 \\
\hline 3 & $-5.5(-14.3,5.6)$ & 0.3 & $1.2(-5.1,7.5)$ & 0.7 & $-7.6(-16.7,1.5)$ & 0.1 & $-7.5(-15.8,0.8)$ & 0.08 & $-5.6(-16.2,5.0)$ & 0.3 & $0.01(-9.5,9.5)$ & 1.0 \\
\hline \multicolumn{13}{|l|}{ SP-AZ-residence ${ }^{a}$} \\
\hline \multicolumn{13}{|l|}{ Urban } \\
\hline Peri-urban & $5.0(-7.7,17.6)$ & 0.4 & $2.1(-6.7,11.0)$ & 0.6 & $2.9(-9.9,15.7)$ & 0.7 & $-1.1(-12.8,10.6)$ & 0.9 & $-8.2(-26.8,10.3)$ & 0.4 & $8.4(-6.1,23.0)$ & 0.3 \\
\hline Rural & $2.7(-8.2,13.6)$ & 0.6 & $2.7(-5.0,10.3)$ & 0.5 & $7.5(-3.5,18.5)$ & 0.2 & $-3.9(-14.0,6.1)$ & 0.4 & $-2.8(-18.7,13.1)$ & 0.7 & $7.7(-4.8,2.2)$ & 0.2 \\
\hline Migrant & $1.2(-19.5,21.9)$ & 0.9 & $7.2(-7.3,21.7)$ & 0.3 & $-5.63(-26.55,15.29)$ & 0.6 & $3.7(-15.43,22.78)$ & 0.7 & $-17.6(-47.8,12.6)$ & 0.3 & $0.5(-23.3,24.2)$ & 1.0 \\
\hline
\end{tabular}


Table 3 Relative antibody responses against P. falciparum antigens at delivery by treatment arm in Madang, PNG, adjusted for confounding and interaction variables (Continued)

\begin{tabular}{|c|c|c|c|c|c|c|c|c|c|c|c|c|}
\hline \multicolumn{13}{|c|}{ SP-AZ-ethnicity ${ }^{a}$} \\
\hline \multicolumn{13}{|c|}{ Madang/Morobe } \\
\hline Sepik & $0.02(-11.0,11.00)$ & 1.0 & $1.9(-5.9,9.6)$ & 0.6 & $5.1(-6.0,16.2)$ & 0.4 & $-4.6(-14.7,5.6)$ & 0.4 & $-1.6(-17.7,14.4)$ & 0.8 & $11.5(-1.1,24.1)$ & 0.07 \\
\hline Highlands & $-1.0(-16.0,14.0)$ & 0.9 & $-1.3(-11.8,9.2)$ & 0.8 & $1.6(-13.6,16.7)$ & 0.8 & $-5.53(-19.4,8.3)$ & 0.4 & $-2.8(-24.7,19.1)$ & 0.8 & $6.7(-10.6,23.9)$ & 0.5 \\
\hline Others & $0.5(-14.2,13.1)$ & 0.9 & $3.3(-6.3,12.9)$ & 0.5 & $6.6(-7.2,20.5)$ & 0.4 & $-3.6(-16.2,9.0)$ & 0.6 & $-1.0(-21.1,19.2)$ & 0.9 & $-3.6(-19.3,12.1)$ & 0.7 \\
\hline
\end{tabular}

Data represented as coefficients and $95 \%$ confidence interval (Multiple linear regression models), $P$-values are also shown

$S P$ sulfadoxine-pyrimethamine, $A Z$ azithromycin, against control group (SP-CQ), $C Q$ chloroquine

${ }^{a}$ Refers to relative antibody responses in intervention group (SP-AZ). A positive coefficient implies an increase of antibody levels. A negative coefficient implies a decrease of antibody levels. Significant associations

$(p<0.05)$ highlighted in bold 


\section{Abbreviations}

SP: Sulfadoxine-pyrimethamine; MIP: Malaria in pregnancy; IPTp: Intermittent preventive therapy during pregnancy; ITN: Insecticide-treated bed nets; PNG: Papua New Guinea; ANC: First antenatal visit; CQ: Chloroquine; AZ: Azithromycin; LMS: Light microscopy; qPCR: Quantitative polymerase chain reaction; IgG: Immunoglobulin G; ELISA: Enzyme-lined immunosorbent assay; les: Infected erythrocytes; PfEMP1: P. falciparum erythrocyte membrane protein 1.

\section{Competing interests}

The authors declare that they have no competing interests.

\section{Authors' contributions}

Conceived and designed the experiment: SJR, GVB, IM Performed the experiments: AT, WH. Analysed the data: AT, SR. Contributed reagents/ materials/analysis tools: AT, SJR, IM, PMS, HU, LMR. Wrote the paper: AT, GVB, SJR. All authors read and approved the final manuscript.

\section{Acknowledgements}

We thank the pregnant women for their participants in the study. We are grateful to Dr. Maria Ome-Kaius, Dr. Regina Wangnapi and Dr. Sarah Hanieh for clinical support, and Dr. Alex Umbers, Dr. Leanne Robinson, Elvin Lufele, Paula Samol, and other staff for laboratory support. We would also like to acknowledge Prof James Beeson and Christine Langer for providing merozoite antigens, MSP 3 and PfRh2, and Prof Robin Anders for merozoite antigen, MSP2.

\section{Funding}

This study was supported by National Health and Medical Research council of Australia awarded to SJR and GVB, Grant number: 10244441. Sample collection was supported in part by Malaria in Pregnancy Consortium, which receives funding from the Bill and Melinda Gates Foundation. The funders had no role in study design, data collection and analysis, decision to publish or preparation of the manuscript.

\section{Author details}

${ }^{1}$ University of Melbourne, Department of Medicine (Royal Melbourne Hospital), Melbourne, VIC, Australia. ${ }^{2}$ Victorian Infectious Diseases Service, The Doherty Institute, Melbourne, VIC, Australia. ${ }^{3}$ Papua New Guinea Institute of Medical Research, Goroka, Papua New Guinea. ${ }^{4}$ The Walter and Eliza Hall Institute of Medical Research, Melbourne, VIC, Australia. ${ }^{5}$ Barcelona Centre for International Health Research (CRESIB), Barcelona, Spain. ${ }^{6}$ The Nossal Institute for Global Health, The University of Melbourne, Melbourne, VIC, Australia.

Received: 3 December 2014 Accepted: 20 May 2015

Published online: 26 May 2015

\section{References}

1. Brabin BJ. An analysis of malaria in pregnancy in Africa. Bull World Health Organ. 1983;61:1005-16.

2. Fried M, Nosten F, Brockman A, Brabin BJ, Duffy PE. Maternal antibodies block malaria. Nature. 1998;395:851-2.

3. Ataíde R, Mwapasa V, Molyneux ME, Meshnick SR, Rogerson SJ. Antibodies that induce phagocytosis of malaria infected erythrocytes: effect of HIV infection and correlation with clinical outcomes. PLoS One. 2011;6:e22491.

4. Feng G, Simpson JA, Chaluluka E, Molyneux ME, Rogerson SJ. Decreasing burden of malaria in pregnancy in Malawian women and its relationship to use of intermittent preventive therapy or bed nets. PLoS One. 2010;5:e12012

5. Aitken EH, Mbewe B, Luntamo M, Kulmala T, Beeson JG, Ashorn P, et al. Antibody to $P$. falciparum in pregnancy varies with intermittent preventive treatment regime and bed net use. PLoS One. 2012;7:e29874.

6. Intermittent preventive treatment with Azithromycin-containing Regimens in pregnant women in Papua New Guinea (IPTp in PNG) [http://clinicaltrials.gov/ show/NCT01136850]

7. Bulmer JN, Rasheed FN, Francis N, Morrison L, Greenwood BM. Placental malaria. I Pathological classification. Histopathology. 1993;22:211-8.

8. Teo A, Hasang W, Randall LM, Feng G, Bell L, Unger H, et al. Decreasing malaria prevalence and its potential consequences for immunity in pregnant women. J Infect Dis. 2014;210:1444-55.
9. Aitken EH, Mbewe B, Luntamo M, Maleta K, Kulmala T, Friso MJ, et al. Antibodies to chondroitin sulfate A-binding infected erythrocytes: dynamics and protection during pregnancy in women receiving intermittent preventive treatment. J Infect Dis. 2010;201:1316-25.

10. Fowkes FJ, McGready R, Cross NJ, Hommel M, Simpson JA, Elliott SR, et al. New insights into acquisition, boosting, and longevity of immunity to malaria in pregnant women. J Infect Dis. 2012;206:1612-21.

11. Ampomah P, Stevenson L, Ofori MF, Barfod L, Hviid L. Kinetics of B cell responses to Plasmodium falciparum erythrocyte membrane protein 1 in Ghanaian women naturally exposed to malaria parasites. J Immunol. 2014;192:5236-44.

12. Osier FH, Feng G, Boyle MJ, Langer C, Zhou J, Richards JS, et al. Opsonic phagocytosis of Plasmodium falciparum merozoites: mechanism in human immunity and a correlate of protection against malaria. BMC Med. 2014;12:108.

13. Staalsoe T, Shulman CE, Dorman EK, Kawuondo K, Marsh K, Hviid L. Intermittent preventive sulfadoxine-pyrimethamine treatment of primigravidae reduces levels of plasma immunoglobulin $\mathrm{G}$, which protects against pregnancy-associated Plasmodium falciparum malaria. Infect Immun. 2004;72:5027-30.

14. Unger HW, Ome-Kaius M, Wangnapi RA, Umbers AJ, Hanieh S, Suen CS, et al. Sulphadoxine-pyrimethamine plus azithromycin for the prevention of low birthweight in Papua New Guinea: a randomised controlled trial. BMC Med. 2015;13:9.

15. Ndyomugyenyi R, Clarke SE, Hutchison CL, Hansen KS, Magnussen P. Efficacy of malaria prevention during pregnancy in an area of low and unstable transmission: an individually-randomised placebo-controlled trial using intermittent preventive treatment and insecticide-treated nets in the Kabale Highlands, southwestern Uganda. Trans R Soc Trop Med Hyg. 2011;105:607-16.

16. Menendez C, Bardaji A, Sigauque B, Romagosa C, Sanz S, Serra-Casas E, et al. A randomized placebo-controlled trial of intermittent preventive treatment in pregnant women in the context of insecticide treated nets delivered through the antenatal clinic. PLoS One. 2008;3:e1934.

\section{Submit your next manuscript to BioMed Central and take full advantage of:}

- Convenient online submission

- Thorough peer review

- No space constraints or color figure charges

- Immediate publication on acceptance

- Inclusion in PubMed, CAS, Scopus and Google Scholar

- Research which is freely available for redistribution 